\title{
Speech Perception in Older Listeners with Normal Hearing: Conditions of Time Alteration, Selective Word Stress, and Length of Sentences
}

\author{
Soojin Cho ${ }^{1}$, Jyaehyoung $\mathrm{Yu}^{2}$, Hyungi Chun², Hyekyung Seo ${ }^{3}$, and Woojae $\mathrm{Han}^{4}$ \\ 1 Department of Speech-Language and Audiology, Nambu University, Gwangju, \\ ${ }^{2}$ Department of Speech Pathology and Audiology, Graduate School, Hallym University, Chuncheon, \\ ${ }^{3}$ Graduate School of Social Welfare, Hallym University, Chuncheon, \\ ${ }^{4}$ Division of Speech Pathology and Audiology, Research Institute of Audiology and Speech Pathology, College of Natural Sciences, \\ Hallym University, Chuncheon, Korea
}

\begin{abstract}
Received March 21, 2014
Revised March 30, 2014

Accepted April 2, 2014
\end{abstract}

Address for correspondence

Woojae Han, PhD

Division of Speech Pathology and

Audiology, Hallym University,

1 Hallimdaehak-gil,

Chuncheon 200-702, Korea

Tel $+82-33-248-2216$

Fax +82-33-256-3420

E-mail woojaehan@hallym.ac.kr
Background and Objectives: Deficits of the aging auditory system negatively affect older listeners in terms of speech communication, resulting in limitations to their social lives. To improve their perceptual skills, the goal of this study was to investigate the effects of time alteration, selective word stress, and varying sentence lengths on the speech perception of older listeners. Subjects and Methods: Seventeen older people with normal hearing were tested for seven conditions of different time-altered sentences (i.e., $\pm 60 \%, \pm 40 \%, \pm 20 \%, 0 \%$ ), two conditions of selective word stress (i.e., no-stress and stress), and three different lengths of sentences (i.e. short, medium, and long) at the most comfortable level for individuals in quiet circumstances. Results: As time compression increased, sentence perception scores decreased statistically. Compared to a natural (or no stress) condition, the selectively stressed words significantly improved the perceptual scores of these older listeners. Long sentences yielded the worst scores under all time-altered conditions. Interestingly, there was a noticeable positive effect for the selective word stress at the $20 \%$ time compression. Conclusions: This pattern of results suggests that a combination of time compression and selective word stress is more effective for understanding speech in older listeners than using the time-expanded condition only.

Korean J Audiol 2014;18(1):28-33

KEY WORDS: Auditory perception · Older speech perception · Time alteration · Selective word stress $\cdot$ Length of spoken words.

\section{Introduction}

The degradation of speech comprehension is one of the characteristics of older listeners (i.e., adults 65 years of age or older), indicating poor temporal and frequency resolutions, especially for complex speech sounds. ${ }^{1)}$ In a noisy environment, ${ }^{2)}$ reverberation listening condition, ${ }^{3)}$ or fast speaking rates, ${ }^{4}$ speech perception ability among those who are older is much worse than for younger listeners. These findings confirm that age-related changes in speech perception result from

This is an Open Access article distributed under the terms of the Creative Commons Attribution Non-Commercial License (http://creativecommons. org/licenses/by-nc/3.0/) which permits unrestricted non-commercial use, distribution, and reproduction in any medium, provided the original work is properly cited. a combination of peripheral and central auditory factors. ${ }^{5)} \mathrm{Al}-$ though there is strong evidence for the importance of peripheral audibility in explaining the poor speech perception of older listeners, ${ }^{6}$ many contemporary researchers argue that agerelated central auditory declines largely affect speech perception in this population. That is, older listeners show much poorer speech perception than their younger adult counterparts even in similar absolute sensitivity. ${ }^{7)}$ Less favorable listening conditions (e.g., less semantic context, the absence of prosodic and syntactic information, increasing the difficulty of lexical selection, and the use of multiple or unfamiliar talkers) and deficits in working memory capacity and inhibitory control are enough to cause age-related differences in speech perception, which may result from declines in both general cognitive abili- 
ties and specialized perceptual mechanisms used for speech communication. ${ }^{5)}$

Fortunately, several lines of research suggest that older listeners can overcome some speech perception difficulties by deploying compensatory central processing. In general, older listeners exhibit performance deficits compared to younger listeners in recognizing time-compressed sentences. ${ }^{4)}$ A study by Gordon-Salant and Fitzgibbons ${ }^{8)}$ also proved that in particular the time compression of consonant parts brings out larger age-related deficits than does time compression of vowels or pauses. Thus, if increasing the speed of a spoken message through time compression is disadvantageous for older listeners, then slowing down the speed of the spoken message should be beneficial to them as a solution. From this point of view, Sommers, et al. ${ }^{9)}$ derived that word recognition scores improved in a time-expanded condition, even with noise. However, prior efforts to enhance speech perception for older listeners by decreasing the presentation rate have been successful in only a few studies. For example, older listeners have shown reduced scores with time expansion of monosyllables because these stimuli may have been too limited in duration to observe any benefits of time expansion. ${ }^{10)}$ A study by Vaughan, et al. ${ }^{11)}$ was also unsuccessful in that temporal distortions of speech in time expansion may be detrimental to speech recognition under noise situation. In Gordon-Salant and Fitzgibbons's study, ${ }^{12)}$ a large number of older listeners showed a significant decline in speech recognition scores in sentence recall with increasing pause duration (or inter-word intervals) although a few older listeners showed a little improvement. One possible reason for this decline in performance may be the disruption in the natural rhythm of the sentences inadvertently created by inserting silence at unnatural junctures between words. Based on current research studies thus far, although articulating speech at a little slower speed is common in early aural rehabilitation, its influence on speech perception of certain pathologic groups is still poorly understood to many clinicians.

On the other hand, another research group addresses overcoming the older listener's perceptual difficulties in terms of word emphasis. Vaughan, et al. ${ }^{11)}$ confirmed that the speech recognition performance of older listeners was improved while increasing consonant gain feature. In the experiment, they increased consonant energy by $10 \mathrm{~dB}$ relative to the energy in the accompanying vowel. As a result, they found that high consonant gain was helpful at the slowest rate under quiet conditions, and there was a positive effect for the consonant gain with noise both at the normal rate and at the moderately slowed rate of speech. ${ }^{11)}$ However, when consonant gain was combined with the largest time expansion in noise, the effects were detrimental to speech recognition. Therefore, further study of more nat- ural gain input is needed, rather than only emphasizing the consonant part.

Memory span, the number of words a person can recall in order immediately after hearing them, increases with age from approximately three words at age three to about seven words for adults. ${ }^{13)}$ In general, the faster a person speaks, the more he or she could place on memory loop before overwriting previous information. However, memory span in older listeners, which is a common measure of their short-term memory ability, largely decreases with age due to intrinsic factors, ${ }^{14)}$ especially at a faster speaking rate. Compared to their younger counterparts, older listeners need more mental energy during effortful listening, showing more errors, slower processing, or forgetting of perceptual tasks. ${ }^{14)}$ Thus, we effectively aid better speech perception skills of older listeners who generally have a limited memory span. Their memory span would benefit from a combination of time alteration and/or selective word stress, to some degree.

The present study evaluates three factors, i.e., time alteration, selective word stress, and different lengths of sentences, among several circumstances that can work to enhance the speech perception of older listeners. We hypothesize that the sentence perception scores would be significantly different among these three main factors, and also expect to find some interactions that may provide a cue to enhance the speech perception ability of older listeners. Meaningful results of the current study would make improved listening conditions available for many older listeners in terms of rehabilitative audiology.

\section{Subjects and Methods}

\section{Subjects}

A group of 17 older listeners between 65 and 85 years old (mean age: 74.08 years; 6 male and 11 female) with normal hearing as a function of age ${ }^{15)}$ were randomly recruited. These participants showed a normal tympanogram and their puretone average for 4 frequencies $(0.5,1,2$, and $4 \mathrm{kHz})$ was 20.31 $\pm 10.1 \mathrm{~dB}$ HL having air-bone gaps no greater than $5 \mathrm{~dB}$ HL. They were also screened using the mini-mental state examination (MMSE-K) ${ }^{16)}$ at 25 and higher scores, which means free of mild cognitive impairment and dementia. They were monolingual Korean speakers. Before starting the experiment, all completed the informed consent form.

\section{Stimuli}

As stimuli, the Korean standard-sentence list for adults ${ }^{17)}$ was modified. The previous eight lists having 10 sentences each were pair-switched in terms of similar word class and sentence structure (list 1 vs. 3 , list 2 vs. 5, list 4 vs. 7, list 6 vs. 8). Final- 
ly, eight new lists were reformed to semantically anomalous sentences. The reason is that the age effect for recognition of time-altered speech is larger for speech materials with reduced linguistic cues, as in random-order phrases from sentences, compared with that observed for meaningful sentences. ${ }^{4)}$ The new lists consisted of varying sentence length of $2-7$ syllables with equal number. In our experiments, sentences of 2-3 syllables, 4-5 syllables, 6-7 syllables were defined as short, medium, and long, respectively. All speech materials were prerecorded by a female talker who is a native speaker of Korean with no pronounced regional dialect at average 4.8 syllables per second, ${ }^{18)}$ and then digitized and stored on compact disc.

To create seven conditions of time compression and expansion, Adobe Audition (ver. 5.0, Adobe Systems Inc., Mountain View, CA, USA) was used. Except for the list 6 having similar mean scores but the highest standard deviation when testing four pilot subjects, we selected seven lists: $60 \%$ compression (list 7)(Fig. 1A), 40\% compression (list 5), 20\% compression (list 3), normal (or $0 \%$ compression/expansion; list 8), 20\% expansion (list 1), 40\% expansion (list 2), 60\% expansion (list 4)(Fig. 1B). Pauses between sentences and between lists were 5 and 7 seconds, respectively. Root mean squ-

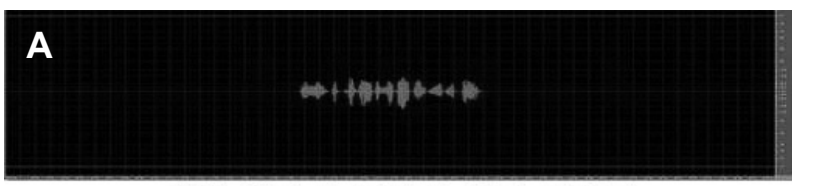

내일 없어서 만나서 열지 갈꺼야

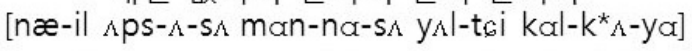
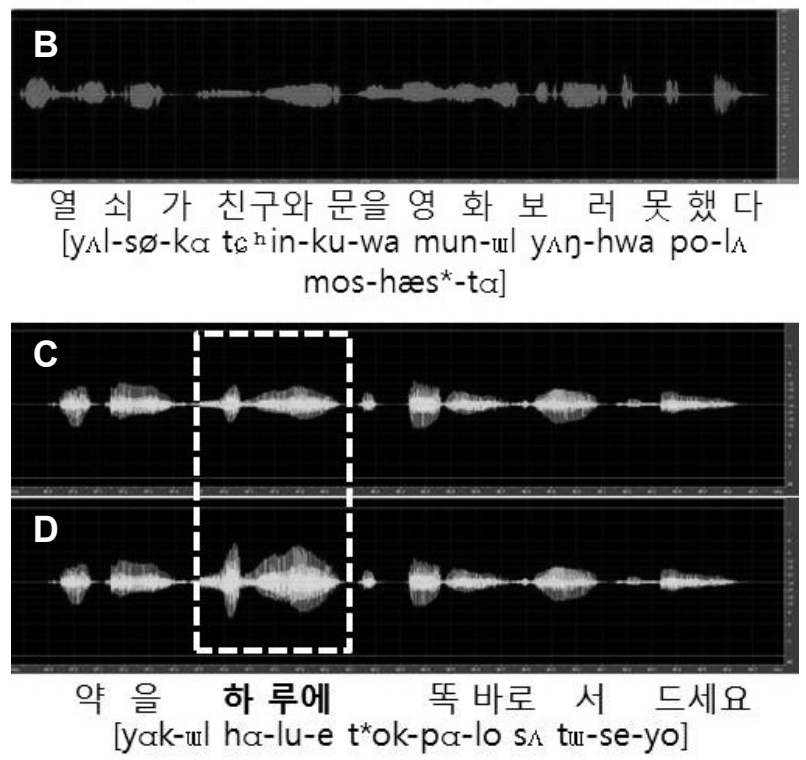

Fig. 1. An example of stimulus in time alteration condition: $60 \%$ compression (A) and $60 \%$ expansion (B). An example of stimulus for the selective word stress condition in normal speech rate: natural (or no stress) (C) and $+6 \mathrm{~dB}$ stress condition (D). International Phonetic Alphabet symbols were displayed for transcribing Korean dialects into English. are was used for adjusting amplitude, but inherent characteristics of words including fundamental frequency were always fixed.

For the selective word stress conditions (i.e., the emphasis on specific syllables in the sentences), 10 words per list were chosen among words without euphonic changes. Since 5-8 $\mathrm{dB}$ increasing was meaningful to enhance speech perception, ${ }^{19)} 6 \mathrm{~dB}$ (or twice the sound pressure level) for the selective word stress was emphasized by using Adobe Audition (Fig. 1C, D). Finally, two versions (stress and no-stress) of the stimuli on compact disc were created.

\section{Procedures}

All audiometric testing took place in a standard doublewalled, sound-treated chamber. We screened pure-tone air and bone conduction thresholds using a Grason-Standler model 61 clinical audiometer with TDH 50 earphones and a Radio Ear B71 bone oscillator. Speech materials were presented from the compact discs through the right side of the earphone (i.e., considering right-ear advantage) controlled by the clinical audiometer at the individual's most comfortable level (MCL). MCL used was 45-60 dB HL.

The subjects were instructed to repeat the sentences exactly as they heard them and to guess the words if they were unsure. While controlling seven different time-altered conditions (e.g., $\pm 60 \%, \pm 40 \%, \pm 20 \%, 0 \%$ ), subjects were first tested at the normal speech rate (or $0 \%$ compression/expansion condition) as the familiar situation, and then tested under the remaining six conditions in a random order. To observe the effect of selective word stress, all participants were tested twice, 2 weeks apart while avoiding possible learning effect.

\section{Data analysis}

The participants repeated the sentences right after listening to them, and scores were analyzed by two examiners using the percentage of the correct total number of syllables in each given sentence. Percent correct obtained from 17 participants for seven time alterations, two word stress conditions, and three different lengths of sentences were compared using a repeated analysis of variance (SPSS ver. 22, IBM Inc., Armonk, NY, USA) to determine the significant difference. Tukey honestly significant difference was used for the post hoc test, and a bonferroni correction was performed for a confidence interval adjustment of significant main effects and interactions. The criterion used for statistical significance was $p<0.05$.

\section{Results}

While means and standard errors for all measures were 
shown in the Table 1, the results of the study showed the following. There were significantly different sentence perception scores for the different time-altered conditions $[\mathrm{F}(6,90)=$ $124.660, p<0.001]$. With the $60 \%$ compressed and $60 \%$ expanded conditions, the scores significantly decreased and increased, respectively (Fig. 2).

There was a significant difference in sentence perception scores under the effect of selective word stress $[F(1,15)=$ $41.527, p<0.001]$. Compared to the natural (or no stress) condition, the scores improved approximately $4 \%$ with the selective word stress in general. There was an interaction between time alteration and the selective word stress $[\mathrm{F}(6,90)=4.644$, $p<0.001]$. Except for only $60 \%$ compression, which is the fastest speech rate, the word stress condition in the other six speech rates showed from only $1 \%$ higher at $40 \%$ expansion to $10.2 \%$ higher at $20 \%$ compression when compared to the nostress condition (Fig. 3). Scores of the word stress condition on $20 \%$ compression were as high as $81.85 \%$ correct, which means similar high scores to no-stress (or natural condition) on $60 \%$ expansion condition only.

Depending on the length of sentences, there was also significant difference $[\mathrm{F}(2,30)=9.174, p<0.001]$. As the length of sentences increased, the scores in general decreased. Due to $2.08 \%$ higher in long sentences than medium sentences at $40 \%$ compressed condition, however, an interaction between time alteration and the length of sentences was revealed $[\mathrm{F}(12$, $180)=4.126, p<0.001$ ] (Fig. 4).

There was no interaction between word selective stress and the length of sentences $[\mathrm{F}(2,30)=0.279, p=0.758]$, and also no interaction among the three factors, i.e., time alteration, word selective stress, and the length of sentences $[F(12,180)=1.637$, $p=0.076]$. There was a significant gender difference, although the gender balance of the participants was not appropriate $[\mathrm{F}$ $(1,15)=4.974, p=0.041]$. Scores of the female subjects [mean: 75.49; standard error (SE): 1.34 ] were approximately 5\% higher than male subjects (mean: 70.46; SE: 1.81) in percent of sentences correct.

\section{Discussion}

As a possible explanation of age-related deficits in speech perception, the sensorineural hearing loss of older listeners, called presbycusis, can result in difficulties for inappropriate central auditory processing by deteriorated input signals as well as the peripheral encoding of input sounds. ${ }^{20)}$ Age-related speech perception difficulties are not highly correlated with the degree and configuration of hearing loss and are often greater than pure-tone thresholds would predict. ${ }^{21)}$ These difficulties are thought to reflect underlying problems in auditory tempo-

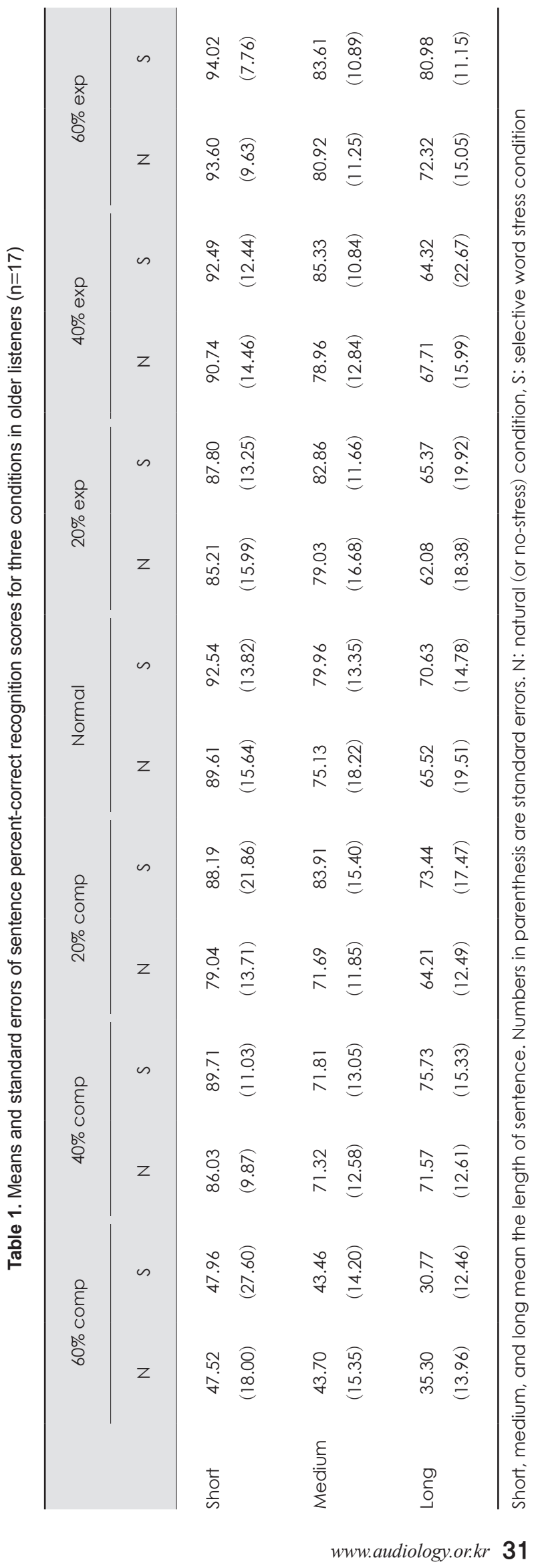




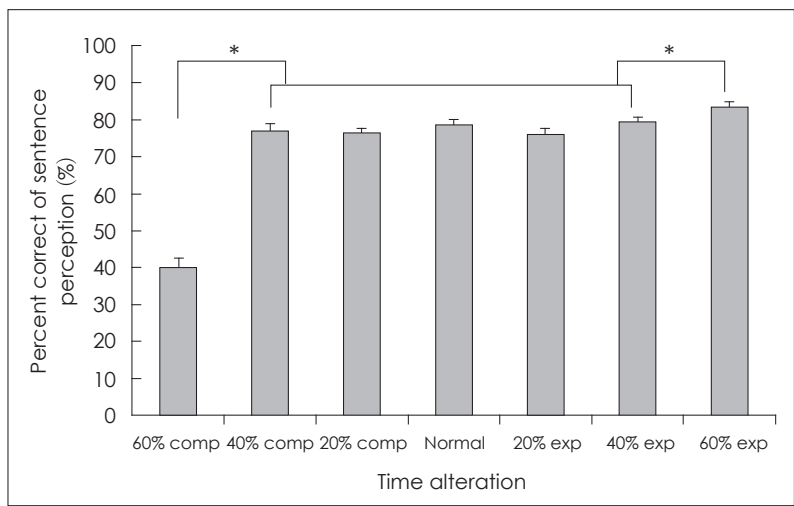

Fig. 2. Mean percent-correct recognition scores and standard errors of the seven time-altered conditions; $60 \%$ comp means $60 \%$ time-compressed condition and $20 \%$ exp means $20 \%$ time-expanded condition. Significant differences in the percent correct are marked with asterisks $(p<0.05)$.

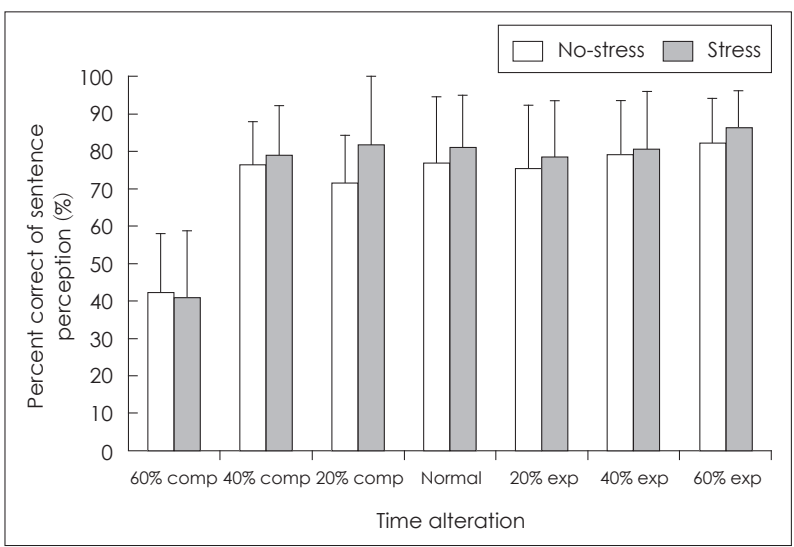

Fig. 3. Mean percent-correct recognition scores and standard errors of the seven time-altered conditions for the selective word stress. No-stress (white bars) means a natural condition while stress (gray bars) means selective word stress condition.

ral processing by older people that may occur at various stages of peripheral and central auditory pathways. ${ }^{22)}$

The present study was designed to help older listeners who suffer from difficulty understanding spoken language in everyday communicative situations in spite of little impaired hearing sensitivity. As we expected, there were significantly different sentence perception scores for the different time-altered conditions. In particular, recognition of time-compressed speech appears to be affected by the temporal characteristics: higher scores in time expansion and lower scores in time compression. These were the same results as those found in many previous studies. Vaughan and Letowski ${ }^{23)}$ showed that older listeners exhibited excessive difficulty recognizing speech delivered at a faster than normal presentation rate. This was primarily attributed to a reduction in overall processing of specific acoustic cues for fast speech. ${ }^{8}$ In addition, older listeners have been shown to have difficulty discriminating changes in the presentation rate of tonal sequences as well as difficulty rec-

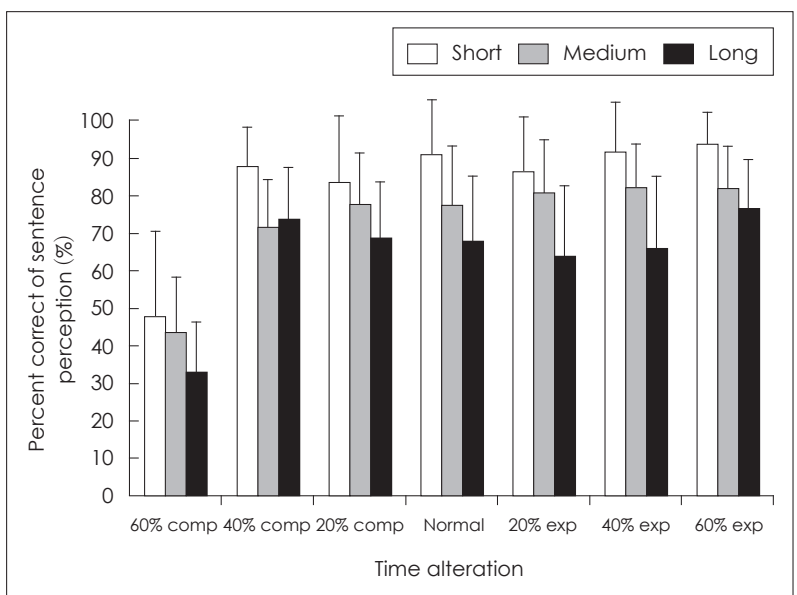

Fig. 4. Mean percent-correct recognition scores and standard errors of the seven time-alteration conditions for the length of sentences. Short (white bars), medium (gray bars), and long (black bars) means the length of sentence.

ognizing the order of items presented in rapid auditory sequences. ${ }^{24)}$ When compared to our previous study with young normal-hearing listeners under the same experimental conditions, ${ }^{25)}$ the older listeners were more adversely affected by increased speech rates than were younger listeners. One interpretation is that the dynamic nature of speech requires rapid processing to keep pace with incoming information for older listeners.

The effect of selective word stress was also significant for perceptual improvement in the older listener population, but not for younger listeners of our previous study. ${ }^{25)}$ Unlike Vaughan, et al.'s ${ }^{11)}$ study which showed poor scores in time expended condition, we obtained successful results in both time compression and expansion in terms of significant percent score enhancement. A significant $10 \%$ higher improvement in speech perception at selective word stress condition even at a slightly faster speed of speech (i.e., $20 \%$ compression) than natural conditions was one of the meaningful findings of our study. This pattern of results suggests that a combination of time compression and selective word stress is more effective for understanding speech for older listeners than using the time expansion only. It also suggests that a complementary approach to designing test batteries that can assess both sensory and cognitive abilities needed for processing speech perception may offer the most appropriate approach for developing therapeutic and rehabilitative interventions to improve perceptual ability in older listeners. We expect that these results will apply to the aural rehabilitation of hearing-impaired listeners as well as presbycusis in further studies.

An effect of sentence length was also observed: longer sentences were much harder for older listeners than shorter sentences. However, no significant finding was observed between 
the two variables, word selective stress and the length of sentences, or among the three variables. One related possibility is that all listeners have difficulty inhibiting the processing of irrelevant information when the target signal itself is difficult to recognize due to random-order words. It was supported that the memory performance of older listeners is highly related to familiar information such as factual, semantic, and autobiographical information. ${ }^{26)}$ Moreover, familiar verbal information is not completely preserved when accessed on the output side (i.e., speech production) rather than the input side, which exhibits significant age-related declines. ${ }^{26)}$

There are limitations of the current study that warrant further research. First, the small sample size $(n=17)$ in the older listeners limited the power of the current analyses to find some interactions among our variables which we hypothesized. A second issue comes from our findings, i.e., a significant positive effect for the selective word stress at the $20 \%$ time compression, whether older listeners who have some degree of sensorineural hearing loss could be applied. Despite of these limitations, the results of the current study have various possible implications for every listening situations and audiological clinic settings.

\section{Conclusion}

Our investigation addressed the efficacy of a combination of time alteration and selective word stress consisting of different lengths of sentences for improving speech perception in older listeners. The principal findings of the current study are:

1) As time compression increased, the sentence perception scores statistically decreased.

2) Compared to a natural condition, the selective word stress significantly improved the perceptual scores of these older listeners.

3) Longer sentences having 6-7 syllables yielded worse scores for older listeners.

4) There was an interesting positive effect between the selective word stress and a slightly faster speed of speech.

\section{Acknowledgments}

The authors would in particular like to thank all the older participants from Chuncheon Dongbu Senior Welfare Center for lending their time and effort to this study.

\section{REFERENCES}

1) Pichora-Fuller MK. Cognitive aging and auditory information processing. Int J Audiol 2003;42 Suppl 2:2S26-32.

2) Plomp R, Mimpen AM. Speech-reception threshold for sentences as a function of age and noise level. J Acoust Soc Am 1979;66:1333-42.

3) Nábělek AK, Robinson PK. Monaural and binaural speech perception in reverberation for listeners of various ages. J Acoust Soc Am
1982;71:1242-8.

4) Wingfield A, Poon LW, Lombardi L, Lowe D. Speed of processing in normal aging: effects of speech rate, linguistic structure, and processing time. J Gerontol 1985;40:579-85.

5) Sommers MS. Age-related changes in spoken word recognition. In: Pisoni DB, Remez RE, editors. The Handbook of Speech Perception. UK, Oxford: Blackwell Publishing;2006. p.469-93.

6) Gates GA, Cooper JC Jr, Kannel WB, Miller NJ. Hearing in the elderly: the Framingham cohort, 1983-1985. Part I. Basic audiometric test results. Ear Hear 1990;11:247-56.

7) Sommers MS, Danielson SM. Inhibitory processes and spoken word recognition in young and older adults: the interaction of lexical competition and semantic context. Psychol Aging 1999;14:458-72.

8) Gordon-Salant S, Fitzgibbons PJ. Sources of age-related recognition difficulty for time-compressed speech. J Speech Lang Hear Res 2001; 44:709-19.

9) Sommers MS, Nygaard LC, Pisoni DB. Stimulus variability and spoken word recognition. I. Effects of variability in speaking rate and overall amplitude. J Acoust Soc Am 1994;96:1314-24.

10) Gordon-Salant S, Fitzgibbons PJ, Friedman SA. Recognition of timecompressed and natural speech with selective temporal enhancements by young and elderly listeners. J Speech Lang Hear Res 2007; 50:1181-93.

11) Vaughan NE, Furukawa I, Balasingam N, Mortz M, Fausti SA. Timeexpanded speech and speech recognition in older adults. J Rehabil Res Dev 2002;39:559-66.

12) Gordon-Salant S, Fitzgibbons PJ. Selected cognitive factors and speech recognition performance among young and elderly listeners. J Speech Lang Hear Res 1997;40:423-31.

13) Hulme C, Muir C. Developmental changes in speech rate and memory span: a causal relationship? Brit J Dev Psychol 1985;3:175-81.

14) Baldwin CL, Ash IK. Impact of sensory acuity on auditory working memory span in young and older adults. Psychol Aging 2011;26:8591.

15) Lee JH, Kim JS, Oh SY, Kim KS, Cho SJ. Effects of age on hearing thresholds for normal adults. Korean J Audiol 2003;7:15-23.

16) Kwon YC, Park JH. Korean version of mini-mental state examination (MMSE-K). Part I: development of the test for the elderly. J Korean Neuropsychiatr Assoc 1989;28:125-35.

17) Jang H, Lee J, Lim D, Lee K, Jeon A, Jung E. Development of Korean standard sentence lists for sentence recognition tests. Audiology 2008;4:161-77.

18) Shin SJ, Shin JC, Yoon MS, Kim DY. Effects of speech rate on the sentence perception of adults with cochlear implantation. Korean J Speech Sci 2006;13:47-58.

19) Picheny MA, Durlach NI, Braida LD. Speaking clearly for the hard of hearing. II: acoustic characteristics of clear and conversational speech. J Speech Hear Res 1986;29:434-46.

20) Humes LE. Speech understanding in the elderly. J Am Acad Audiol 1996;7:161-7.

21) Crandell CC, Henoch MA, Dunkerson KA. A review of speech perception and aging: some implications for aural rehabilitation. J Acad Rehabil Audiol 1991;24:121-32.

22) Schneider BA, Daneman M, Murphy DR. Speech comprehension difficulties in older adults: cognitive slowing or age-related changes in hearing? Psychol Aging 2005;20:261-71.

23) Vaughan NE, Letowski T. Effects of age, speech rate, and type of test on temporal auditory processing. J Speech Lang Hear Res 1997;40: 1192-200.

24) Fitzgibbons PJ, Gordon-Salant S, Friedman SA. Effects of age and sequence presentation rate on temporal order recognition. J Acoust Soc Am 2006;120:991-9.

25) Han W, Yu J, Cho S. Perception of time-altered sentences and selective word stress by normal-hearing listeners. J Acoust Soc Korea 2013;32:430-7.

26) Burke DM, Mackay DG. Memory, language, and ageing. Philos Trans R Soc Lond B Biol Sci 1997;352:1845-56. 